\title{
A Lifecycle Simulation Framework for Production Systems
}

\author{
Masaru Nakano $^{1}$, Shigetoshi Noritake ${ }^{1}$, Toshio Ohashi ${ }^{2}$, \\ 1 Toyota Central R\&D Laboratories., Inc., Nagakute, Aichi, 480-1192, \\ Japan \\ URL: http://www.tytlabs.co.jp/eindex.html \\ 2 Toyota Motor Corporation, Toyota, Aichi, 471-8571, Japan \\ URL: http://www.toyota.co.jp/en/
}

\begin{abstract}
The prediction of market behavior is helpful for a manufacturing enterprise to build efficient production systems, but unfortunately these predictions are usually not very reliable. Subsequently, development of more flexible production systems is important to adapt to changing markets but basically cause a higher cost than less flexible ones. This paper proposes a lifecycle simulation framework for production systems by combining the two topics. The simulation structure has several template libraries consisting of many scenarios or patterns of market behaviors, product lineups, production lines, and reconfiguration policies. The framework is initially described for a factory, and afterwards expanded for a global production network.
\end{abstract}

\section{Introduction}

A modern manufacturing enterprise producing and selling consumer products has a complex business architecture, integrating many processes from marketing research to the product and production development process to supply chain and sales management (Kubota, Sato and Nakano [1]). The entire enterprise has a more complicated decision making process related to production system design in need of constant improvement to stay competitive in the world. Prediction of market behavior is very helpful to build efficient production systems, but unfortunately, demand forecasting is not overly reliable. Development of more flexible production systems is important to adapt to turbulent, fast-changing markets and basically requires higher costs than less flexible ones. Many studies have been done for either market analysis or the flexibility, adaptability and changeability of production systems. Studies integrating both the market demands and the flexibility provided by the production system are required to design production systems for the future. These

Please use the following format when citing this chapter:

Nakano, M., Noritake, S. and Ohashi, T., 2008, in IFIP International Federation for Information Processing, Volume 257, Lean Business Systems and Beyond, Tomasz Koch, ed.; (Boston: Springer), pp. 327-335. 
topics are very related to the studies on lifecycle-oriented design or the flexibility for production systems (See [2], [3], [4]).

The literature regarding manufacturing flexibility was classified by Toni and Tochia [2]. While they provided many references for the process and volume flexibility in production, no studies are introduced for the flexibility related to a change in the product itself. Autich and Barbian [3] proposed a project management technique for lifecycle oriented design by evaluating the flexibility. Burkner et. al. [5] evaluated the flexibility for volume changes from the view of risk by simulation in combination with market scenario analysis. This paper discusses the flexibility for not only product volume but also changes in the product itself from the view of production system lifecycle and proposes a framework for lifecycle simulation.

\section{Lifecycle Oriented Design for Production Systems}

There are two ways how production systems can strategically adjust to market changes: product engineering and sales, or production engineering as shown in Figure 1. The production engineering and management may be considered on a factory level (In-factory), a supply chain level including multiple factories (Crossfactory), or an enterprise level (Outsource). The cross-factory and outsource levels have more flexibility than the in-factory level.

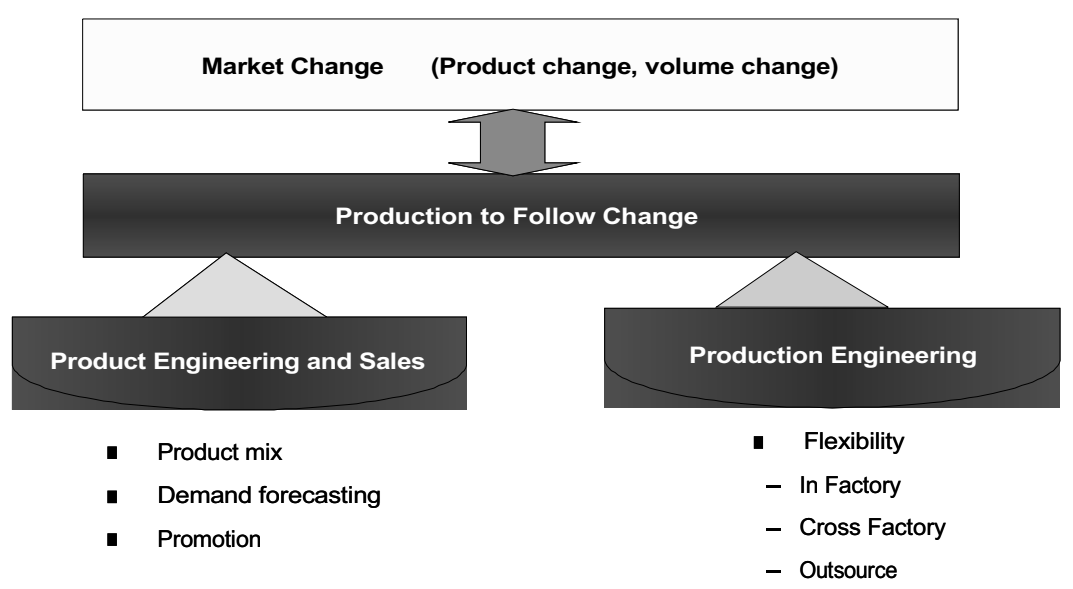

Fig. 1. Production Flexibility and Market Change

The lifecycle-oriented design has engineering processes from product ramp-up to end of life stages as shown in Figure 2. The flexibility for production has two main phases of the reconfiguration: The first phase is the investment during production preparation and start-up phase, and the second phase is the efficiency improvements during regular production. Lifecycle simulation should be used only after the start-up phase is completed because the start-up phase is too complex and has too much 
manually involvement to be simulated. Large changes in the number of product features, production volume or product mix may require a similar engineering process as with the start-up stage. However, if the revisions to the original production plan are relatively small, the stages can be simulated by providing basic patterns of revisions.

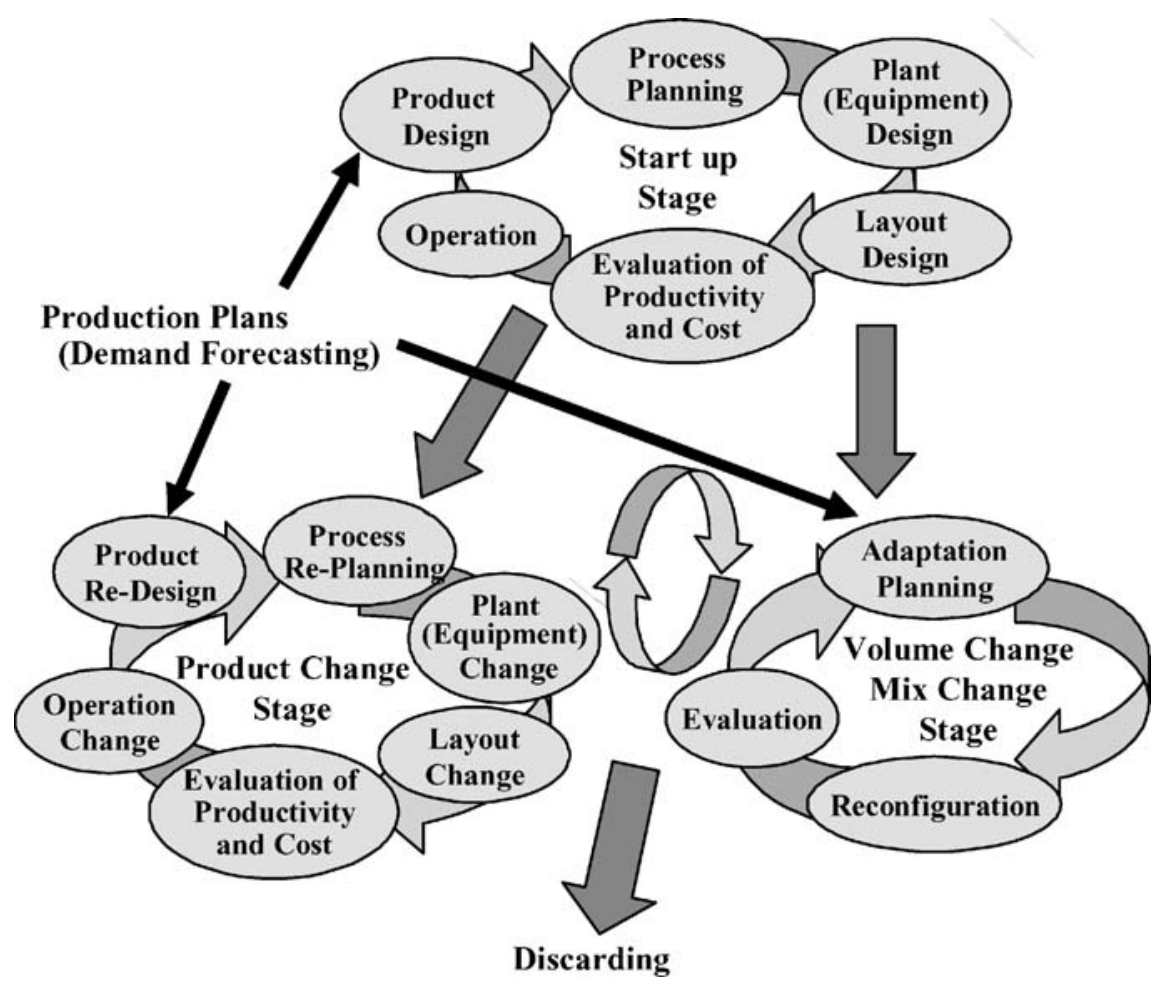

Fig. 2. Engineering Process for Production System Lifecycle

\section{Lifecycle Oriented Simulation}

A lifecycle simulation structure is proposed for a single factory as shown in Figure 3. The program modules can be computed using an iterative routine with the orders as shown by the arrows in Figure 3 or alternatively using a distributed architecture as explained in Nakano, et al. [7]. The templates and the program modules are explained as follows:

The Product Scenario Templates specify the timing and change rates in features to be launched for product change.

The Factory Scenario Templates define the limit of changes in demand or the number and complexity of product features for small changes. Reconfiguration 
patterns are also provided for large changes. Reuse patterns of machines, process planning, and machine configuration are included in the detailed case.

The Market Scenario Templates provides market demands as time series. These are generated using historic data or market prediction simulation.

The Initial settings navigate the user to initially set decision variables such as the initial production volume. Initial production lines and the sets of the efficiency curves are also typical decision variables used to optimize the production systems during the lifecycle.

The Product Lifecycle Scenario Generator generate triggers which happen at a predetermined time and initialize a product change, a specification change, or a change in the expected production volume.

The Production System Reconfiguration Scenario Cost Estimator estimates the cost of the reconfiguration.

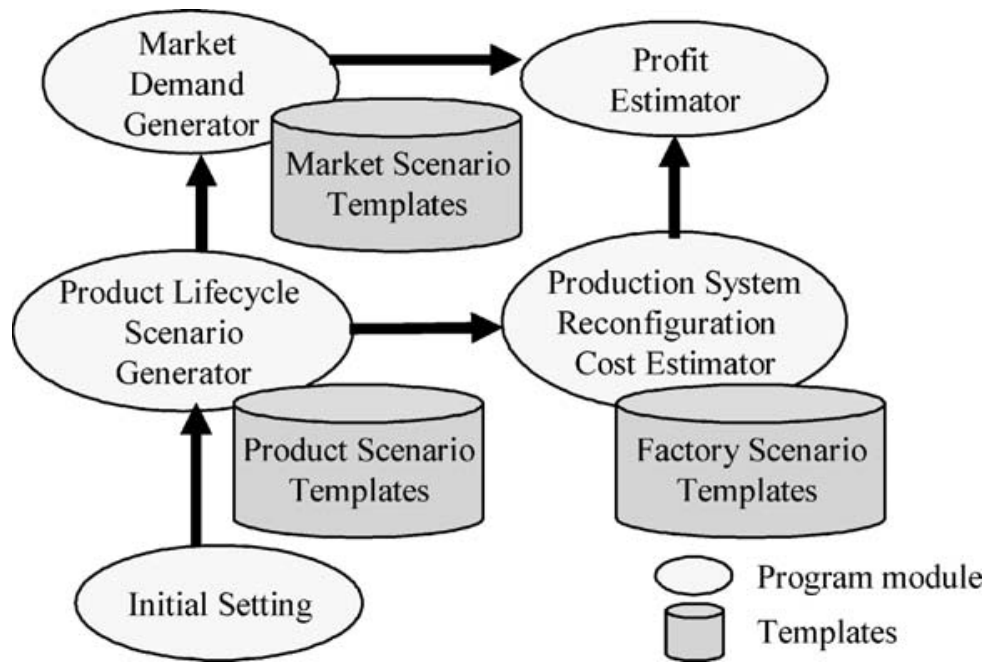

Fig. 3. A Simulation Structure for a Single Factory

The Market Demand Generator generates the market demands as a time series with fluctuations based on a selected scenario template as defined by the Market Scenario Template. A Monte Carlo simulation generates many market fluctuation scenarios with many different variations.

The Profit Estimator calculates the lifecycle cost of the production, and estimates the profit obtained from the sales at the factory level. The outcome is a distribution of the EBIT (earnings before interest and tax). The simulation period includes a few changes for each product model. If an accurate simulation is required, a production system simulator (for example, see [8]) can be employed as a part of the estimator. 


\section{Usage of the Lifecycle Oriented Simulation}

\subsection{Aim of Simulation}

The following questions can be answered with the use of the lifecycle oriented simulation:

1) How much does the flexibility of a machine or a structure of a production system affect the lifecycle cost and profit? The information provides a guideline to develop or reuse machines and production systems.

2) How far can market fluctuation be allowed while still guarantee a profit? The information can provide the goal of product planning and sales promotion.

3) Which strategy is the best for a reconfiguration?

\subsection{Reconfiguration Cost Calculation}

A reconfiguration may be necessary to adapt to a large change in demand beyond the limit of the flexibility of the production system or to a product change. The following three methods are considered to calculate the reconfiguration cost.

\section{a) Experience based functional with flexibility relations}

The efficiency of a production line is related through a curve to the production volume. A reconfiguration scenario for volume changes is made by jumping from one curve to another depending on the details of the changes as shown in Figure 5. The volume flexibility influences the timing of reconfiguration. The more flexible a production line is, the less often a reconfiguration is expected. If the change cost is estimated based on experience, the total lifecycle cost can be evaluated. The timing of reconfiguration can also be used to adapt for product changes. The reconfiguration cost is calculated according to the extent of product change. The more flexible a production line is, the more initial investment and the less change cost can be expected in the case of automated processing lines. Figure 6 shows these relations.

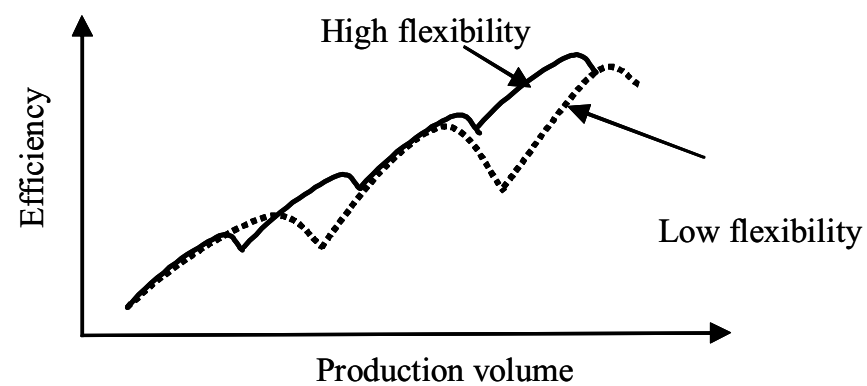

Fig. 4. Production Volume and Efficiencies for Different Flexibilities 


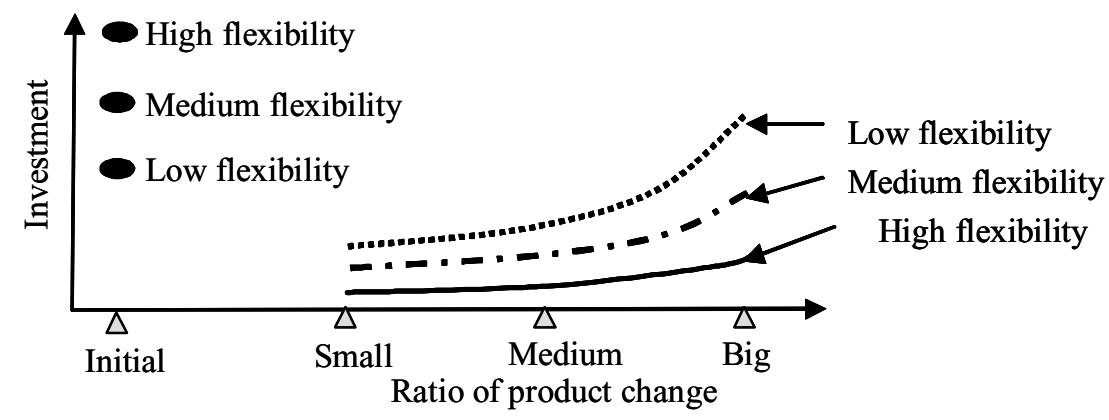

Fig. 5. Product Change and Required Investment for Different Flexibilities

\section{b) Experience based table with flexibility relations}

A table can be prepared based on experience to determine the reconfiguration cost by summing up the change costs of machines and the costs for relocating workers. A very simple example is shown in Table 1. An initial investment cost for different flexibility is evaluated in the same way as the reconfiguration cost.

Table 1. A Simple Example of Reconfiguration Cost for Different Flexibilities

\begin{tabular}{|c|l|l|l|l|}
\hline \multicolumn{2}{|c|}{} & \multicolumn{3}{|c|}{ Production Flexibility } \\
\cline { 3 - 6 } & Low & Medium & High \\
\hline \multirow{3}{*}{ Volume Change } & Low & Low & Low & Low \\
\cline { 2 - 5 } & Medium & High & Medium & Low \\
\cline { 2 - 5 } & High & High & High & High \\
\hline \multirow{3}{*}{ Mix Change } & Low & Low & Low & Low \\
\cline { 2 - 5 } & Medium & High & Medium & Low \\
\cline { 2 - 5 } & High & High & High & Medium \\
\hline \multirow{3}{*}{ Product Change } & Low & Medium & Medium & Low \\
\cline { 2 - 5 } & Medium & High & Medium & Medium \\
\cline { 2 - 5 } & High & High & High & High \\
\hline
\end{tabular}

\section{c) Automatic generation}

An appropriate production process is optimized if product features and machines are provided. If the process is almost fixed and the change is relatively small, a semiautomatic or a rough-cut redesign technique can be employed. The rough-cut redesign process goes from product design to process planning, machine redesign, and layout redesign, to evaluation of the productivity and cost. Additional templates such as production processes and machines are also needed. See Okuda et al. [6] for a related study. 


\subsection{How to use the Simulation for a Simple Example}

This section shows the steps to use the simulation for simple examples. Assume a simple example consisting of a product and a production line with a related efficiency curve. The initial production demand and the production efficiency to meet the demand are given. Choose some demand curves in the Templates of Market Demand, samples of which are shown in Figure 7.

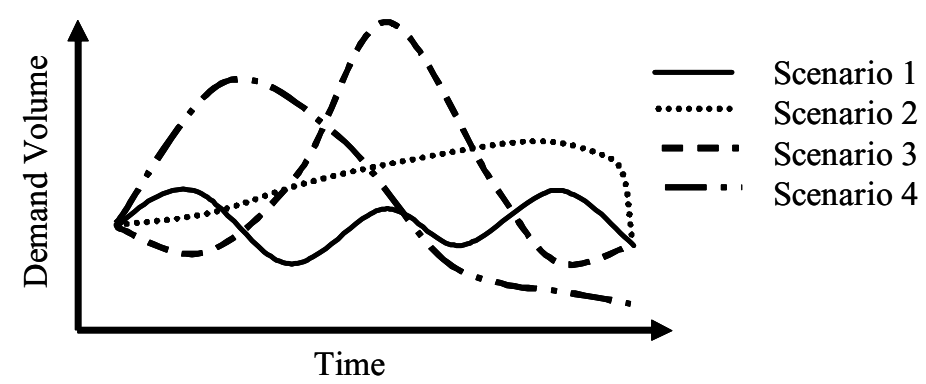

Fig. 6. Sample Demand Curves

For example, assume each of the four curves has $25 \%$ possibility. When the volume in demand exceeds the predetermined extent, the Production System Scenario Generator invests to increase the production capacity, as for example a duplicate of the line. The production flexibility for reconfiguration is shown in Table 1. Select from three levels in flexibility to be used as a decision variable. The redesign process is periodically initiated through a product change in the product model cycles. The Profit Estimator calculates the lifecycle cost by averaging the results from multiple replicas of simulation with different demand scenarios under the selected possibilities. The risk can be estimated in terms of value at risk (VAR).

\section{Extension of the Framework to Evaluate Global Supply Networks}

A global production network with multiple factories has more flexibility and adaptability than a single production line. If different factories have different efficiency curves due to different characteristics such as automation rates or possible mixture rates, you can select appropriate production lines at appropriate locations to adapt to the volume or product change. The idea is extended to integrate a lifecycle simulation with a supply network simulation including outsourcing. If a business component is not a core source, you can outsource it to a supplier in order to optimize the flexibility and adaptability in the supply network. The topic can also be extended to optimize a product lineup to maximize profits. Therefore this research topic is not only related to production system design but also enterprise design in 
terms of mid-term lifecycle management. Therefore, this paper proposes a technical framework of modeling, evaluating and planning a production system lifecycle model in the manufacturing enterprise. Figure 8 shows the extension of the simulation model for a global supply chain model.

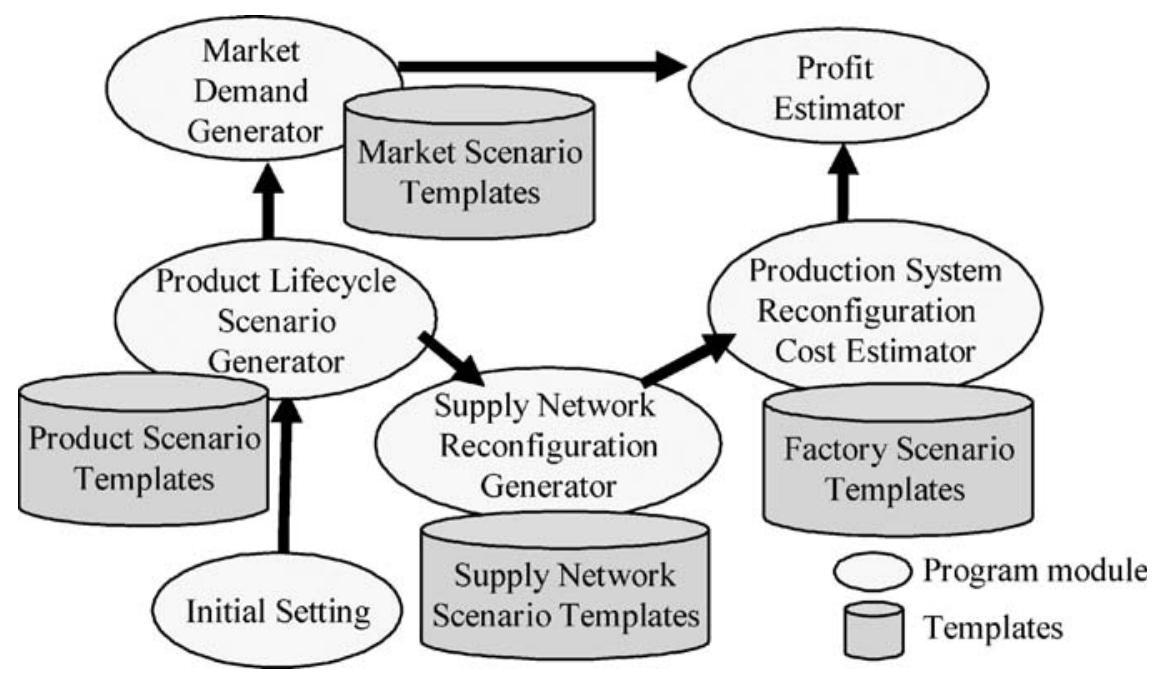

Fig. 7. A Simulation Structure for a Global Network

The following new modules are added to the simulation of a single factory to extend the model to a global supply chain simulation:

The Supply Network Scenario Templates includes various supply network configurations. Economies of scale can be considered for outsourcing, merging, collaboration, and vertical or horizontal integration.

The Supply Network Reconfiguration Generator selects appropriate production systems in appropriate factories in a globally distributed network, decomposes the estimated production volumes to the appropriate production systems, and reconfigures the supply network with a scenario tree where each node in the tree represents a possible plan of the supply network configuration.

\section{Conclusion}

The paper proposes a framework for lifecycle simulation for production systems and shows the extension of the production system simulation to entire supply networks. The study was done in the international collaboration project LicoPro between EU and Japan. While simple experiments are studied in the project (see [5], [6]), this paper describes the basic idea for further discussion. The authors believe that the discussion of the leanness in production management with a viewpoint of the 
lifecycle of entire production systems within this paper will stimulate the interests of researchers in the academic society.

\section{Acknowledgments}

The study was supported by the IMS program under Ministry of Economy, Trade and Industry of Japan.

\section{References}

1. F. Kubota, S. Sato, M. Nakano, Enterprise Modeling and Simulation Integrating Manufacturing System Design and Supply Chain, IEEE Int. Conf. on System, Man, and Cybernetics, pp.511-515 (1999).

2. A. De Toni and S. Tonchia, Manufacturing flexibility: a literature review, Int. J. of Production Research, Vol.36, No.6, pp.1587-1617 (1998).

3. J. C. Autich and P. Barbian, Production Projects - Designing and Operating Lifecycle-Oriented and Flexibility-Optimized Production Systems as a Project, Int. J. of Production Research, Vol.42, No.17, pp.3589-3601 (2004).

4. G. Schuh, C. Schleyer and F. Zohm, Planning Methodology for LifecycleOriented, Flexible Production Systems, The 1st Conf. on Changeable, Agile Reconfigurable and Virtual Production (CARV) (2005).

5. S. Burkner, M. Schmitt, J. Roscher and M. Friese, Methods for Flexibility Evaluation in the Automotive Industry, The CARV (2005).

6. T. Okuda, K. Ohashi, H. Matsuoka, S. Noritake, H. Nagase, M. Nakayama, K. Furusawa, T. Muraki, S. Chino, S. Fujita, K. Wada, Y. Sano, M. Nakano, H. Kubota, M. Anan, T. Ohashi, F. Kimura, Lifecycle-Oriented Design of Flexible and Agile production System (LicoPro), IMS Int. Forum2004, pp.626-633 (2004).

7. M. Nakano, F. Kubota, Y. Inamori and K. Mitsuyuki., Method and Tool to Visualize and Analyze Business Processes, Knowledge and Skill Chains in Engineering and Manufacturing, edited by E. Arai (Springer), pp.177-184 (2004).

8. M. Nakano, N. Sugiura, M. Tanaka, and T. Kuno, ROPSII: Agent-Oriented Manufacturing Simulator on the Basis of Robot Simulator, Proc. of JAPAN-U.S.A. Symposium on Flexible Automation, pp.201-208 (1994). 